\title{
Fluvastatin and Atorvastatin Affect Calcium Homeostasis of Rat Skeletal Muscle Fibers in Vivo and in Vitro by Impairing the Sarcoplasmic Reticulum/Mitochondria $\mathrm{Ca}^{2+}$-Release System
}

\author{
Antonella Liantonio, Viviana Giannuzzi, Valentina Cippone, Giulia Maria Camerino, \\ Sabata Pierno, and Diana Conte Camerino \\ Unità di Farmacologia, Dipartimento Farmacobiologico, Facoltà di Farmacia, Università di Bari, Bari, Italy \\ Received December 7, 2006; accepted February 6, 2007
}

\begin{abstract}
The mechanism by which the 3-hydroxy-3-methyl-glutaryl-CoA reductase inhibitors (statins) induce skeletal muscle injury is still under debate. By using fura-2 cytofluorimetry on intact extensor digitorum longus muscle fibers, here we provided the first evidence that 2 months in vivo chronic treatment of rats with fluvastatin ( 5 and $20 \mathrm{mg} \mathrm{kg}^{-1}$ ) and atorvastatin (5 and $10 \mathrm{mg}$ $\mathrm{kg}^{-1}$ ) caused an alteration of calcium homeostasis. All treated animals showed a significant increase of resting cytosolic calcium $\left[\mathrm{Ca}^{2+}\right]_{\mathrm{i}}$, up to $60 \%$ with the higher fluvastatin dose and up to $20 \%$ with the other treatments. The $\left[\mathrm{Ca}^{2+}\right]_{\mathrm{i}}$ rise induced by statin administration was not due to an increase of sarcolemmal permeability to calcium. Furthermore, the treatments reduced
\end{abstract}

caffeine responsiveness. In vitro application of fluvastatin caused changes of $\left[\mathrm{Ca}^{2+}\right]_{\mathrm{i}}$, resembling the effect obtained after the in vivo administration. Indeed, fluvastatin produced a shift of mechanical threshold for contraction toward negative potentials and an increase of resting $\left[\mathrm{Ca}^{2+}\right]_{\mathrm{i}}$. By using ruthenium red and cyclosporine $A$, we determined the sequence of the statininduced $\mathrm{Ca}^{2+}$ release mechanism. Mitochondria appeared as the cellular structure responsible for the earlier event leading to a subsequent large sarcoplasmic reticulum $\mathrm{Ca}^{2+}$ release. In conclusion, we suggest that calcium homeostasis alteration may be a crucial event for myotoxicity induced by this widely used class of hypolipidemic drugs.
Hydroxymethylglutaryl-coenzyme A (HMG-CoA) reductase inhibitors, also known as statins, are the most useful agents for treatment of hypercholesterolemia. By blocking the rate-limiting step catalyzed by this enzyme in the mevalonate pathway, de novo synthesis of cholesterol is prevented, and low-density lipoprotein cholesterol uptake into cells is promoted (Goldstein and Brown, 1990). The different statins currently available (simvastatin, atorvastatin, lovastatin, pravastatin, fluvastatin, rosuvastatin) are generally well tolerated in patients. However, side effects may arise in skeletal muscle, ranging from transient increase in creatine kinase, muscle pain, and cramps to myositis and potentially lifethreatening rhabdomyolysis (Bellosta et al., 2004; Rosenson, 2004). Despite the numerous studies describing myopathy in animals and humans, the molecular mechanism of statininduced myotoxicity has not been completely elucidated. A

This study was supported by Italian "Ministero dell'Istruzione, dell'Università e della Ricerca” (FIRB RBAU015E9T) (to D.C.C.).

Article, publication date, and citation information can be found at http://jpet.aspetjournals.org.

doi:10.1124/jpet.106.118331. variety of hypotheses have been formulated to explain such toxicity, including impairment of glycoprotein synthesis in the muscle membrane as well as reduction of ubiquinone concentration in mitochondria, causing severe deficits in energy metabolism (Evans and Rees, 2002).

Interestingly, it was also proposed that an alteration of structures involved in $\mathrm{Ca}^{2+}$ homeostasis could play a pivotal role in producing myocyte injury. After 2 to 3 months of chronic treatment of rats with simvastatin, the voltage threshold for contraction (mechanical threshold, MT), a calcium-sensitive index of excitation-contraction coupling, was shifted toward more negative potentials in fast-twitch muscle fibers (Pierno et al., 1999), an effect that is compatible with an increase of resting cytosolic calcium concentration $\left(\left[\mathrm{Ca}^{2+}\right]_{\mathrm{i}}\right)$. Moreover, in vitro studies showed a statin-induced elevation of $\left[\mathrm{Ca}^{2+}\right]_{i}$ and suggested a possible interference of the drug with intracellular $\mathrm{Ca}^{2+}$ stores (Nakahara et al., 1994; Inoue et al., 2003; Sirvent et al., 2005), which in turn might be responsible of cell damages via activation of $\mathrm{Ca}^{2+}$ dependent proteolytic enzymes (Sacher et al., 2005). It is noteworthy that all statin-induced effects on skeletal muscle

ABBREVIATIONS: HMG-CoA, 3-hydroxy-methyl-glutaryl coenzyme A; EDL, extensor digitorum longus; [Ca $\left.{ }^{2+}\right]_{i}$, intracellular calcium concentration; MT, mechanical threshold; $\mathrm{gCl}$, resting chloride conductance; SR, sarcoplasmic reticulum; RyR, ryanodine receptor; PTP, permeability transition pore; RR, ruthenium red; CsA, cyclosporine A; SOCE, store-operated calcium entry; ANOVA, analysis of variance. 
are strictly dependent on their lipophilicity, showing highly hydrophilic pravastatin, and no muscle toxicity, even at high doses (Nakahara et al., 1994; Pierno et al., 1995, 1999). Recently, a multidisciplinary study aimed to the identification of the cellular and tissue targets of a chronic treatment with newer powerful and lipophilic statins, such as fluvastatin and atorvastatin, on rat skeletal muscle was conducted (Pierno et al., 2006). Both statins resulted more potently than simvastatin in producing skeletal muscle injury. Indeed, although no histological changes were observed on muscle fibers, both statin treatments produced a significant negative shift of the potentials of MT at which fibers contracted. In addition, statins altered muscle excitability by reducing resting chloride conductance $(\mathrm{gCl})$, the electrical parameter sustained by the voltage-gated chloride channel CLC-1 critical for the maintenance of membrane stability. Because CLC-1 is negatively regulated by the $\mathrm{Ca}^{2+}$-dependent protein kinase C (De Luca et al., 1998; Rosenbohm et al., 1999), the observed reduction of $\mathrm{gCl}$ supports the hypothesis that statin administration could effectively interfere with calcium-handling mechanism.

Based on the above findings, we performed a 2-month in vivo administration with fluvastatin and atorvastatin to rats and evaluated ex vivo the effect of drug treatment on resting $\left[\mathrm{Ca}^{2+}\right]_{\mathrm{i}}$ by fura- 2 cytofluorimetry on tendon to tendon intact fibers of extensor digitorum longus (EDL) muscle. In parallel, to further elucidate the statin-induced $\mathrm{Ca}^{2+}$ release mechanism, we characterized the statin cellular effects after acute in vitro application.

Our data showed that both statins caused a sustained increase in cytosolic $\mathrm{Ca}^{2+}$ levels by interfering with intracellular stores, such as mitochondria and sarcoplasmic reticulum. Considering the crucial role of resting calcium ions in skeletal muscle function and maintenance, our findings contribute to a better understanding of the mechanism responsible for cramps, myalgia, and other muscular side effects induced by this widely used class of hypolipidemic drugs.

\section{Materials and Methods}

Animals Care and in Vivo Drug Treatment. Animal care and in vivo drug treatment were approved by the Italian Health Department and the Institutional Animal Care and Use Committee according to Italian law (DL 116/92) and the European Community Directive (86/609/EEC). Male Wistar rats (Charles River Laboratories, Calco Como, Italy) initially weighing 300 to $350 \mathrm{~g}$ were used. The animals were housed individually in rat appropriate cages and fed with a commercial rodent chow (approximately $30 \mathrm{~g}$ day $^{-1}$, 4RF21; Charles River Laboratories) and tap water ad libitum. Rooms were maintained at a constant temperature $\left(22-24^{\circ} \mathrm{C}\right)$ and exposed to a light cycle of $12 \mathrm{~h} \mathrm{day}^{-1}$ (8:00 AM-8:00 PM). The animals were subdivided into six experimental groups as follows. The first group (nine rats) was chronically administered with $5 \mathrm{mg} \mathrm{kg}{ }^{-1}$ day $^{-1}$ fluvastatin, the second group (six rats) was administered with $20 \mathrm{mg}$ $\mathrm{kg}^{-1}$ day $^{-1}$ fluvastatin, the third group (six rats) was administered with $5 \mathrm{mg} \mathrm{kg}^{-1}$ day $^{-1}$ atorvastatin, the fourth group (10 rats) was administered with $10 \mathrm{mg} \mathrm{kg}{ }^{-1}$ day $^{-1}$ atorvastatin, the fifth group (six rats) was administered with only the vehicle (aqueous methylcellulose, CMC) used to dissolve the drugs, and the sixth group (seven rats), an untreated control group. During the treatment period, all animals showed normal body weight gain and seemed to be in good health, with the exception of rats chronically treated with high doses of fluvastatin $\left(20 \mathrm{mg} \mathrm{kg}^{-1}\right.$ ) (for details see Pierno et al., 2006). The doses of drugs were chosen based on data present in the literature regarding human and rodents, as reported previously (Pierno et al., 2006). Fluvastatin and atorvastatin dissolved in CMC $(0.5 \%)$ suspension were administered orally via an esophageal cannula once a day for 2 months. For each rat, the weight-related dose was formulated so that the maximal volume of drug-containing suspension was $1 \mathrm{ml}$. Because the results obtained from the two control groups (untreated and CMC-treated rats) were similar, we have combined and showed data as unique control.

In Vivo Study: Determination of the Forelimb Muscle Strength. The forelimb muscle force of control and treated rats was evaluated before and every week until the end of the treatment by means of a grip strength meter (Columbus Instruments, Columbus, $\mathrm{OH}$ ). Five rats per group (five rats treated with $5 \mathrm{mg} / \mathrm{kg}$ fluvastatin, five rats treated with $20 \mathrm{mg} / \mathrm{kg}$ fluvastatin, five rats treated with 5 $\mathrm{mg} / \mathrm{kg}$ atorvastatin, five rats treated with $10 \mathrm{mg} / \mathrm{kg}$ atorvastatin, and five control rats) were analyzed. For this measure, the rats were allowed to grasp a triangular ring connected to a force transducer and then gently pulled away until the grip was broken. The transducer saved the force value at this point as a measure of the maximal resistance; the animal can develop with its forelimbs (De Luca et al., 2003). Five measures (one to five) were taken from each animal within $3 \mathrm{~min}$, and each value was normalized with respect to the weight of the animal. We calculated the difference between the fifth measure and the first measure in each animal as a muscle fatigability index. The mean values ( \pm S.E.M.) of this difference of control and of treated rats were then analyzed for significance (by ANOVA test).

Dissection of Native Muscle Fibers. For ex vivo and in vitro experiments, EDL muscles were removed from the animal under deep urethane anesthesia ( $1.2 \mathrm{~g} / \mathrm{kg}$ body weight). Soon after the surgery, the rats that were still anesthetized were euthanized by anesthetic overdose. EDL muscles of the different groups of treated rats and of control rats were pinned in a dissecting dish containing $95 \% \mathrm{O}_{2} / 5 \% \mathrm{CO}_{2}$-gassed normal physiological solution at room temperature $\left(22^{\circ} \mathrm{C}\right)$ for further dissection. Small bundles of 10 to 15 fibers arranged in a single layer were dissected lengthwise, tendon to tendon, with the use of microscissors, as described elsewhere (Fraysse et al., 2003).

Fura-2 Fluorescence Measurements in Intact Muscle Fibers. Calcium measurements were performed using the membranepermeant $\mathrm{Ca}^{2+}$ indicator fura-2 acetoxymethyl ester (Invitrogen, Pero, Milano, Italy). Loading of muscle fibers was performed for $2 \mathrm{~h}$ at $25^{\circ} \mathrm{C}$ in normal physiological solution containing $5 \mu \mathrm{M}$ fura-2 acetoxymethyl ester mixed to $0.05 \%$ (v/v) Pluronic F-127 (Invitrogen). After loading, muscle fibers were washed with normal physiological solution and mounted in a modified RC-27NE experimental chamber (Warner Instrument Inc., Hamden, CT) on the stage of an inverted Eclipse TE300 microscope (Nikon, Tokyo, Japan) with a $40 \times$ Plan-Fluor objective (Nikon). The mean sarcomere length was set to 2.5 to $2.7 \mu \mathrm{m}$. Fluorescence measurements were made using a QuantiCell 900 integrated imaging system (Visitech International Ltd, Sunderland, UK) as described previously (Fraysse et al., 2003, 2004).

During experiments, pairs of background subtracted images of fura-2 fluorescence $(510 \mathrm{~nm})$ after excitation at 340 and $380 \mathrm{~nm}$ were acquired, and ratiometric images $(340 / 380 \mathrm{~nm})$ were calculated for each muscle fiber of the preparation using QC2000 software. Subsequently fluorescence ratio values were converted to the resting cytosolic calcium $\left[\mathrm{Ca}^{2+}\right]$ (in nanomolars) after a calibration procedure using the equation: $\left[\mathrm{Ca}^{2+}\right]_{\mathrm{i}}=\left(R-R_{\text {min }}\right) /\left(R_{\max }-R\right) \times K_{\mathrm{D}} \times \beta$, where $R$ is the ratio of fluorescence excited at $340 \mathrm{~nm}$ to that excited at 380 $\mathrm{nm} ; K_{\mathrm{D}}$ is affinity constant of fura- 2 for calcium, which was taken as $145 \mathrm{nM}$ (Molecular Probes); and $\beta$ is a parameter according to Grynkiewicz et al. (1985) and was determined experimentally in situ in ionomycin-permeabilized muscle fibers as described previously (Fraysse et al., 2003). $R_{\min }$ and $R_{\max }$ were determined in muscle fibers incubated in $\mathrm{Ca}^{2+}$-free normal physiological solution containing $10 \mathrm{mM}$ EGTA and in normal physiological solution, respectively. 
Determination of Sarcolemmal Permeability to Divalent Cations. The manganese quench technique was used to estimate the sarcolemmal permeability to divalent cations. $\mathrm{Mn}^{2+}$ enters via the same routes as $\mathrm{Ca}^{2+}$ but accumulates inside the cell. As $\mathrm{Mn}^{2+}$ quenches the fluorescence of fura-2, the reduction of the intensity of fura-2 fluorescence can be used as an indicator of the time integral of $\mathrm{Mn}^{2+}$ influx (Parekh and Penner, 1997). Muscle preparations were perfused for $2 \mathrm{~min}$ with normal physiological solution containing 0.5 $\mathrm{mM} \mathrm{Mn}{ }^{2+}$ as a surrogate of $\mathrm{CaCl}_{2}$ (quenching solution). To measure the $\mathrm{Mn}^{2+}$ influx through the store-operated $\mathrm{Ca}^{2+}$ channels, a proper protocol for SOCE activation was used (Kurebayashi and Ogawa, 2001; Zhao et al., 2005, 2006). Particularly, the fibers were perfused with $10 \mu \mathrm{M}$ thapsigargin and $40 \mathrm{mM}$ caffeine for $15 \mathrm{~min}$ in zero $\mathrm{Ca}^{2+}$ extracellular solution to induce sarcoplasmic reticulum (SR) $\mathrm{Ca}^{2+}$

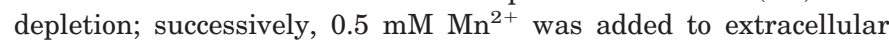
solution. During the quenching protocol, the fluorescence of fura-2 excited at $360 \mathrm{~nm}$ was acquired at $1 \mathrm{~Hz}$.

MT for Contraction. Soon after the removal from the rat, EDL muscles tied at the end of each tendon were placed on a glass rod located in a $25-\mathrm{ml}$ muscle bath chamber maintained at $30^{\circ} \mathrm{C}$ and continuously perfused with $95 \% \mathrm{O}_{2} / 5 \% \mathrm{CO}_{2}$-gassed normal physiological solution. The MT for contraction was determined using a two-microelectrode point voltage-clamp method in the presence of 3 $\mu \mathrm{M}$ tetrodotoxin, as described previously (Pierno et al., 2006). Depolarizing current pulses of increasing duration $(5-500 \mathrm{~ms})$ were given repetitively at a rate of $0.3 \mathrm{~Hz}$, whereas the impaled fibers were viewed continuously with a stereomicroscope ( $100 \times$ magnification). The command voltage was increased until contraction was just visible and the threshold membrane potential at this point was read from a digital sample-and-hold voltmeter. The mean threshold membrane potential (millivolt) \pm S.E.M. ( $n$ fibers) was plotted as a function of the pulse duration $t$ (millisecond), and the relationship was fit using the equation $V(t)=\left[H-R \times \exp \left(-t / \tau_{R}\right)\right] /\left[1-\exp \left(-t / \tau_{R}\right)\right]$, where $H$ is the holding potential $(-90 \mathrm{mV}), R$ (millivolt) is the rheobase voltage, and $\tau_{R}$ (millisecond) is the time constant to reach $R$. The MT values were expressed as the fitted $R$ parameter along with the mean \pm S.E. that was determined from the variance-covariance matrix in the nonlinear least-squares fitting algorithm.

Solution and Chemical Compounds. The normal physiological solution contained $148 \mathrm{mM} \mathrm{NaCl}, 4.5 \mathrm{mM} \mathrm{KCl}, 2.5 \mathrm{mM} \mathrm{CaCl}_{2}, 1 \mathrm{mM}$ $\mathrm{MgCl}_{2}, 0.44 \mathrm{mM} \mathrm{NaH}_{2} \mathrm{PO}_{4}, 12 \mathrm{mM} \mathrm{NaHCO}$, and $5.5 \mathrm{mM}$ glucose. The $\mathrm{pH}$ of all solutions was adjusted to 7.3 to 7.4 by bubbling them with $95 \%$ $\mathrm{O}_{2} / 5 \% \mathrm{CO}_{2}$. The calcium free-solution had the same composition of the normal physiological solution, with the exception that $\mathrm{CaCl}_{2}$ was omitted and $10 \mathrm{mM}$ EGTA was added. The quenching solution had the same composition, with the exception that $0.5 \mathrm{mM} \mathrm{MnCl}_{2}$ was substituted for $\mathrm{CaCl}_{2}$. All chemicals cited above and ionomycin, caffeine, ruthenium red, cyclosporine A, and thapsigargin were purchased from Sigma (St. Louis, MO).

Commercially available fluvastatin (Lescol, fluvastatin sodium, A.I.C. number 029163021; Novartis Novartis, Basel, Switzerland) and atorvastatin (Torvast, atorvastatin calcium, A.I.C. number 033007042; Pfizer Ireland Pharmaceuticals, County Cork, Ireland) in CMC (0.5\%) suspension were used for in vivo chronic treatment to rats. For in vitro experiments, fluvastatin sodium salt purchased from Calbiochem was used.

Statistical Analysis. The ANOVA followed by Bonferroni's $t$ test was used to evaluate statistical differences between treated and control groups. For the in vitro studies, significance levels were calculated using Student's unpaired $t$ test.

\section{Results}

\section{In Vivo Study}

Effects of in Vivo Chronic Treatment with Fluvastatin and Atorvastatin on the Activity-Dependent Change of Forelimb Muscle Strength. We have measured the forelimb muscle strength in control and in all treated groups of rats. In each animal, we have normalized the forelimb strength measured at different time points to body weight to minimize the influence of the body weight gain. At the first analysis, no differences were found in the normalized force between control and all of the rats treated with the different drugs. In particular, the forelimb force measured at the 8 th week of treatment in $5 \mathrm{mg} \mathrm{kg}^{-1} \mathrm{day}^{-1}$ fluvastatin, and $20 \mathrm{mg} \mathrm{kg}^{-1} \mathrm{day}^{-1}$ fluvastatin was $2.20 \pm 0.07 \mathrm{~g} / \mathrm{g}(n=5)$ and $2.49 \pm 0.09 \mathrm{~g} / \mathrm{g}(n=5)$ with respect to the control value of $2.24 \pm 0.07 \mathrm{~g} / \mathrm{g}(n=5)$. However, when we considered the difference between the first and the fifth measure read after $3 \mathrm{~min}$ from the first one, we found that this value was strongly decreased in $20 \mathrm{mg} \mathrm{kg}^{-1}$ day $^{-1}$ fluvastatin-treated rats. Moreover, the decrease was more pronounced for longer duration of treatment (Fig. 1). No difference was found in the rats treated with atorvastatin at both dosages (data not shown). Thus, we found that fluvastatin at high doses was able to significantly increase muscle fatigability in terms of reduced force production due to prolonged activity.

\section{Ex Vivo Studies}

Effects of in Vivo Chronic Treatment with Fluvastatin and Atorvastatin on Skeletal Muscle Calcium Homeostasis. Visible light microscopic changes and voltagedependent calcium release. As useful indexes of the health state of the muscle fibers (Fraysse et al., 2004; Dulhunty, 1992), we first investigated the effect of the in vivo statin chronic treatment on the gross morphologic fibers aspect and on the excitation-calcium release coupling. To this purpose, we examined skeletal muscle fibers samples by visible light microscopy and exposed them to a depolarizing high potassium solution.

As illustrated in Fig. 2 (top), there was no light microscopic changes in EDL muscle fibers of rats treated with $5 \mathrm{mg} \mathrm{kg}^{-1}$

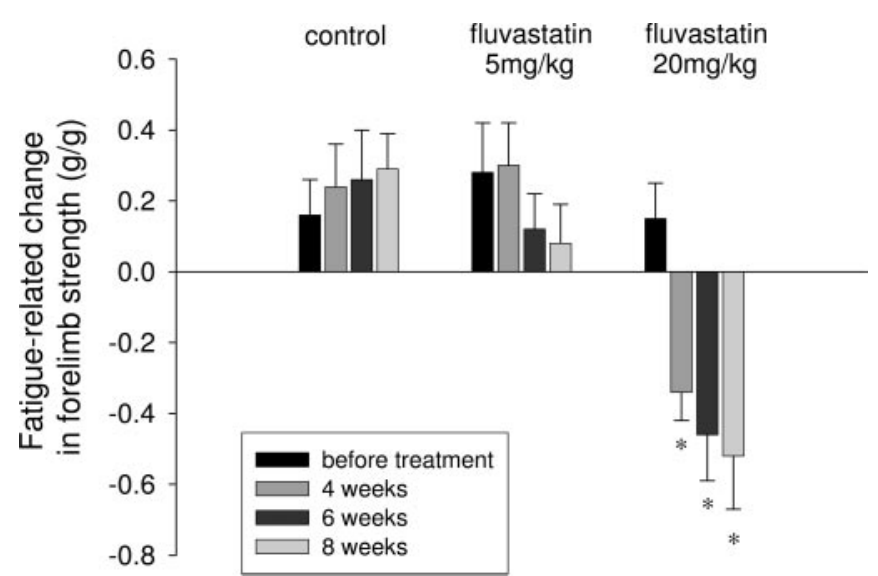

Fig. 1. Effects of in vivo chronic treatment with fluvastatin on the fatigue-related change of the forelimb muscle strength. The forelimb strength has been measured as described under Materials and Methods. In each control and fluvastatin-treated rat, the forelimb strength was determined five times within $3 \mathrm{~min}$, and the difference between the fifth and the first measure has been plotted as an index of muscle fatigability. Each bar is the mean value ( \pm S.E.M.) measured from five animals of each experimental group (controls, 5 and $20 \mathrm{mg} \mathrm{kg}^{-1}$ day $^{-1}$ fluvastatin) and represents the change of normalized forelimb force measured at different time points (before treatment and after 4 to 6 to 8 weeks of treatment). The ANOVA test for multiple comparison shows statistical differences in the $20 \mathrm{mg} \mathrm{kg}^{-1}$ day $^{-1}$ fluvastatin-treated group $(F=6.64, P<0.001)$. Bonferroni's $t$ test showed significant differences with respect to the mean value measured before treatment ( $*, P<0.02$ or less). 

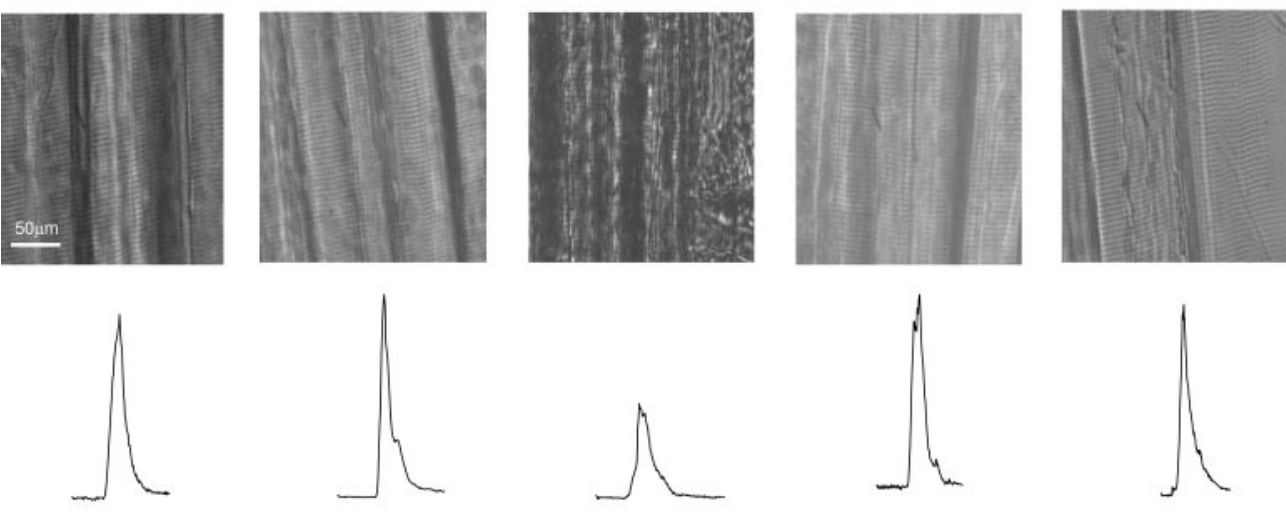

$\frac{100 \mathrm{nM}}{20 \mathrm{sec}}$ day $^{-1}$ fluvastatin and atorvastatin at both dosages compared with control rats. In contrast, fluvastatin-related muscle of rats treated at the dose of $20 \mathrm{mg} \mathrm{kg}{ }^{-1}$ day $^{-1}$ showed a compromised muscle fiber integrity characterized by a remarkable collapse and disarray of sarcomeres. This structural change was accompanied by the alteration of the voltage-dependent $\mathrm{Ca}^{2+}$ release. Indeed, the amplitude of the $\mathrm{Ca}^{2+}$ transient induced by $100 \mathrm{mM} \mathrm{K}{ }^{+}$-depolarizing solution showed by this group of treated animals was reduced by approximately 55\% with respect to the control rats (Fig. 2, bottom).

Measurements of resting $\left[\mathrm{Ca}^{2+}\right]_{i}$. In line with previous studies (Fraysse et al., 2003, 2006), EDL fast-twitch skeletal muscle fibers of adult control rats showed a value of resting $\left[\mathrm{Ca}^{2+}\right]_{\mathrm{i}}$ of $26.9 \pm 0.8 \mathrm{nM}$. Both in vivo fluvastatin and atorvastatin administration induced a significant increase of $\left[\mathrm{Ca}^{2+}\right]_{\mathrm{i}}$ in all of the treated groups, although the effect was more important for animals treated with $20 \mathrm{mg} \mathrm{kg}^{-1}$ day $^{-1}$ fluvastatin (Fig. 3). Indeed, the resting $\left[\mathrm{Ca}^{2+}\right]_{\mathrm{i}}$ was $31 \pm 0.8$ and $43 \pm 2.3 \mathrm{nM}$ in rats treated with $5 \mathrm{mg} \mathrm{kg}^{-1}$ day $^{-1}$ fluvastatin and $20 \mathrm{mg} \mathrm{kg}^{-1}$ day $^{-1}$ fluvastatin, respectively. In the case of atorvastatin, the maximal effect was already observed with the lower dose, reaching a $20 \%$ increase of $\left[\mathrm{Ca}^{2+}\right]_{i}$.

Sarcolemmal permeability to divalent cations. We used the $\mathrm{Mn}^{2+}$-quenching technique to assess the possibility that resting calcium increase in muscle fibers of treated rats, in particular, in $20 \mathrm{mg} \mathrm{kg}{ }^{-1}$ day $^{-1}$ fluvastatin-treated animals, could be related to a greater calcium influx. As it can be seen in Fig. 4A, no modification of sarcolemmal permeability to divalent cations was observed after atorvastatin in vivo treatment. The mean quench rate of $3.06 \pm 0.17$ and $3.20 \pm$ $0.22 \% \mathrm{~min}^{-1}$ for 5 and $10 \mathrm{mg} \mathrm{kg}{ }^{-1}$ day $^{-1}$ atorvastatintreated fibers completely overlapped the value of control rats $\left(3.5 \pm 0.15 \% \mathrm{~min}^{-1}\right)$. Interestingly, although in vivo low dose of fluvastatin did not alter the $\mathrm{Mn}^{2+}$ quench rate, it was drastically reduced to a value that was approximately $60 \%$ lower than that of control rats after the chronic treatment with $20 \mathrm{mg} \mathrm{kg}^{-1} \mathrm{day}^{-1}$ fluvastatin. All together, these data indicate that changes in muscle resting $\left[\mathrm{Ca}^{2+}\right]_{\mathrm{i}}$ observed after the in vivo chronic treatment with fluvastatin and atorvastatin are not due to an increase in the sarcolemmal cationic permeability.

Caffeine-induced calcium release. Because the resting
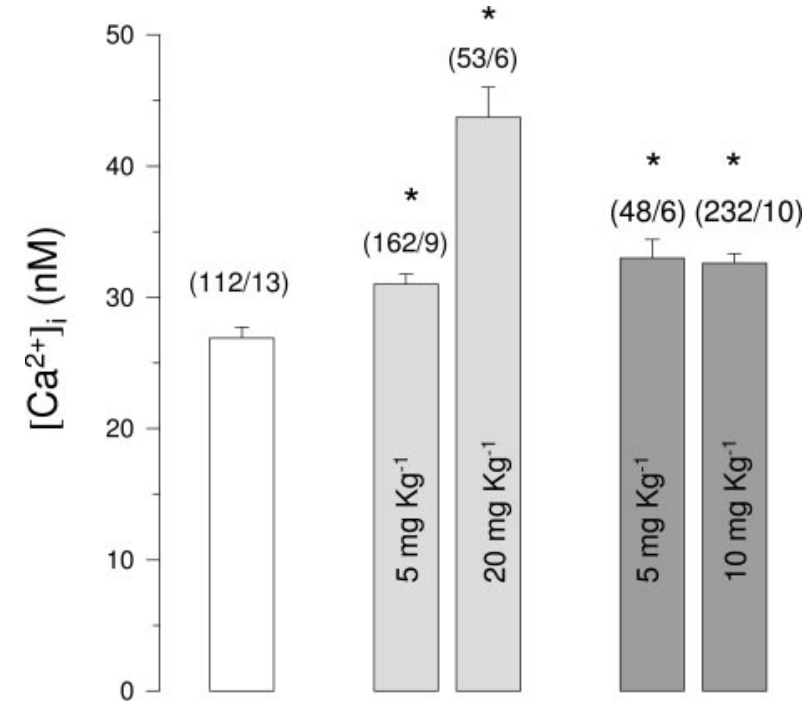

\section{control}

Fig. 2. Effect of the in vivo chronic treatment with fluvastatin and atorvastatin on the gross morphologic fibers aspect and on the excitation-calcium release coupling. Top, visible light microscopic representative images of skeletal muscle fibers samples for each indicated group of rats. Bottom, representative traces for each indicated group of rats showing the $\mathrm{Ca}^{2+}$ transient induced by the application of depolarizing $100 \mathrm{mM} \mathrm{K}^{+}$solution.
Fig. 3. Resting $\left[\mathrm{Ca}^{2+}\right]_{\mathrm{I}}$ measured in EDL muscle fibers from rats chronically treated with fluvastatin ( 5 and $20 \mathrm{mg} \mathrm{kg}^{-1} \mathrm{day}^{-1}$ ) and atorvastatin (5 and $10 \mathrm{mg} \mathrm{kg}^{-1} \mathrm{day}^{-1}$ ) and from control rats. Each bar is the mean \pm S.E.M. from $n$ muscle fibers and $N$ animals $(n / N$ indicated above each bar). Statistical analysis by ANOVA showed significant differences in $\left[\mathrm{Ca}^{2+}\right]_{\mathrm{i}}(F=22, n$ fibers $-k$ groups $=602, P<0.005)$. Bonferroni's $t$ test showed significant differences between all treated groups and the control group $(P<0.005)$.

$\left[\mathrm{Ca}^{2+}\right]_{\mathrm{i}}$ increase was not related to an increased resting permeability of sarcolemma, we investigated whether the statininduced $\left[\mathrm{Ca}^{2+}\right]_{\mathrm{i}}$ increase may be due to an internal calcium store depletion. To evaluate the SR functionality, we exposed muscle fibers to caffeine, which induces a calcium release from SR via activation of ryanodine receptors (RyR) (Rios and Pizarro, 1991). Because the maximal amplitude value of caffeine-induced calcium transient in rat EDL intact muscle fibers was obtained by application of $40 \mathrm{mM}$ caffeine (Fraysse et al., 2003), we determined the caffeine responsiveness of the different animal groups at this dose.

Compared with control rats, a slight amplitude reduction of the calcium transient induced by $40 \mathrm{mM}$ caffeine was observed after treatment with $5 \mathrm{mg} \mathrm{kg}{ }^{-1}$ day $^{-1}$ fluvastatin 
A

control $n=58$

Fluvastatin $5 \mathrm{mg} \mathrm{Kg}^{-1}$ $\mathrm{n}=60$

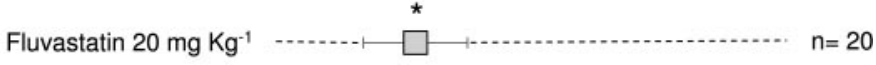

Atorvastatin $5 \mathrm{mg} \mathrm{Kg}^{-1}$

$\mathrm{n}=97$

Atorvastatin $10 \mathrm{mg} \mathrm{Kg}^{-1}$

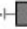

$\mathrm{n}=79$

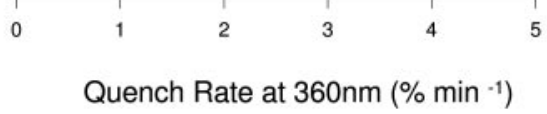

B

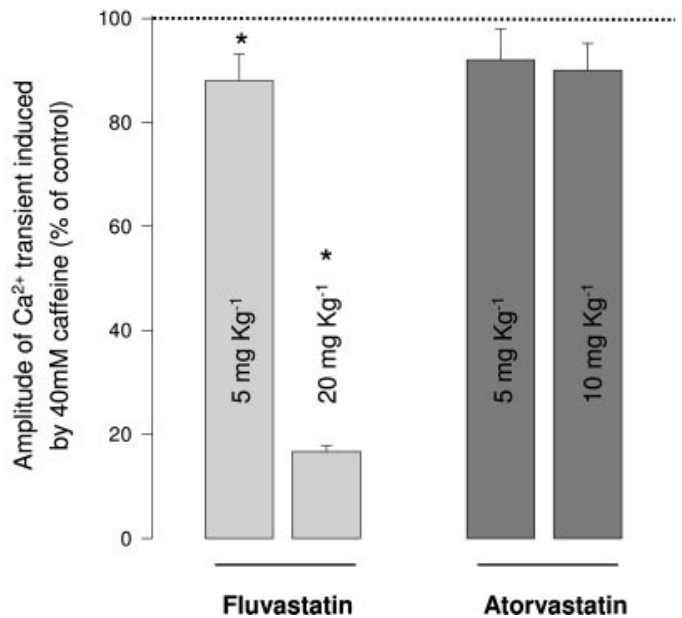

Fig. 4. Effect of in vivo chronic treatment with fluvastatin and atorvastatin on sarcolemma permeability to calcium ions and on caffeine responsiveness. A, the sarcolemma permeability to $\mathrm{Ca}^{2+}$ was evaluated by measuring the quench rate of fura- 2 fluorescence induced by $\mathrm{Mn}^{2+}$ influx. Each square point corresponds to the mean \pm S.E.M. value of quench rates measured in the indicated numbers of muscle fibers of at least three animals. Statistical analysis by ANOVA showed significant differences in quench rate $(F=7, n$ fibers $-k$ groups $=309, P<0.005)$. Bonferroni's $t$ test showed a significant difference between the $20 \mathrm{mg} \mathrm{kg}{ }^{-1}$ day $^{-1}$ fluvastatin-treated group and the control group $(P<0.005)$. B, the caffeine responsiveness was determined by evaluating the amplitude of the calcium transient after in vitro application of $40 \mathrm{mM}$ caffeine for each animal group (25-50 fibers from at least three animals). The mean value of the caffeine-induced calcium transient for each treated animal group has been normalized to the correspondent mean value of the control rats. Thus, each bar represents the normalized percentage of the amplitude of caffeine-induced calcium transient. Statistical analysis by ANOVA showed significant differences in quench rate $(F=58, n$ fibers $-k$ groups $=155, P<0.005)$. Bonferroni's $t$ test showed significant differences between the $5 \mathrm{mg} \mathrm{kg}^{-1}$ day $^{-1}$ fluvastatin-treated group and the 20 $\mathrm{mg} \mathrm{kg}^{-1}$ day $^{-1}$ fluvastatin-treated group with respect the control group $(P<0.005)$.

and atorvastatin at both doses. The lowering of the responsiveness to caffeine was more marked in $20 \mathrm{mg} \mathrm{kg}^{-1}$ day $^{-1}$ fluvastatin-treated rats, showing a $80 \%$ reduction of the calcium transient amplitude (Fig. 4B).

Effect of in Vitro Application of Fluvastatin on Calcium Homeostasis of Skeletal Muscle Fibers. To gain insight into the cellular mechanism of statin-induced calcium handling alteration, we evaluated the drug effect after in vitro acute application on rat skeletal muscle fibers. Based on the results obtained with the in vivo statins administration, we focused our attention on fluvastatin that produced the most evident alteration of the calcium homeostasis in treated rat muscle fibers.

Mechanical threshold and resting $\left[\mathrm{Ca}^{2+}\right]_{i}$. We previously demonstrated that muscle fibers from fluvastatin-treated rats needed less depolarization to contract showing a rheobase voltage $(R)$ shift of 3 to $4 \mathrm{mV}$ toward more negative potentials compared with control rats (Pierno et al., 2006). Accordingly, the in vitro application of fluvastatin on EDL muscle produced a negative shift of the voltage threshold for contraction. Particularly, the effect was observed already after application of $50 \mu \mathrm{M}$ fluvastatin that shifted $R$ of approximately $2 \mathrm{mV}$ toward a more negative potential with respect to the control value (data not shown) and became statistically significant at higher concentration. Indeed, as shown in Fig. 5, $200 \mu \mathrm{M}$ fluvastatin produced a marked shift of strength-duration curve at each pulse duration. The $R$ value obtained by the fit of the experimental points were $-66.0 \pm 0.15$ and $-69.5 \pm 0.25 \mathrm{mV}$ in the absence and presence of $200 \mu \mathrm{M}$ fluvastatin, respectively.

In parallel, micromolar acute application of fluvastatin triggered a sustained rise of $\left[\mathrm{Ca}^{2+}\right]_{\mathrm{i}}$ in fura-2-loaded skeletal muscle fibers (Fig. 6A). Interestingly, the $\mathrm{Ca}^{2+}$ transient induced by $200 \mu \mathrm{M}$ fluvastatin exhibited a peculiar time course, characterized by a slow-rising phase (lasting $15 \mathrm{~min}$ ) followed by a more rapid and large increase of $\left[\mathrm{Ca}^{2+}\right]_{\mathrm{i}}(\mathrm{Fig}$. 6A). Particularly, fluvastatin enhanced cytosolic calcium level from $28.0 \pm 1.8 \mathrm{nM}$ to a plateau value of $124 \pm 20 \mathrm{nM}$, which reached $20 \mathrm{~min}$ after the onset of drug application. When the drug was removed, the fluvastatin-triggered $\mathrm{Ca}^{2+}$ increase slowly (over $15 \mathrm{~min}$ ) returned close to the basal resting $\left[\mathrm{Ca}^{2+}\right]_{\mathrm{i}}$ (data not shown). As can be appreciated from the concentration-response relationship curve (Fig. 6B), such marked increase of $\left[\mathrm{Ca}^{2+}\right]_{i}$ was observed for the dosage $\geq 200$ $\mu \mathrm{M}$. For the dosage below $200 \mu \mathrm{M}$, fluvastatin effect was characterized by only a slow-rising phase responsible for a

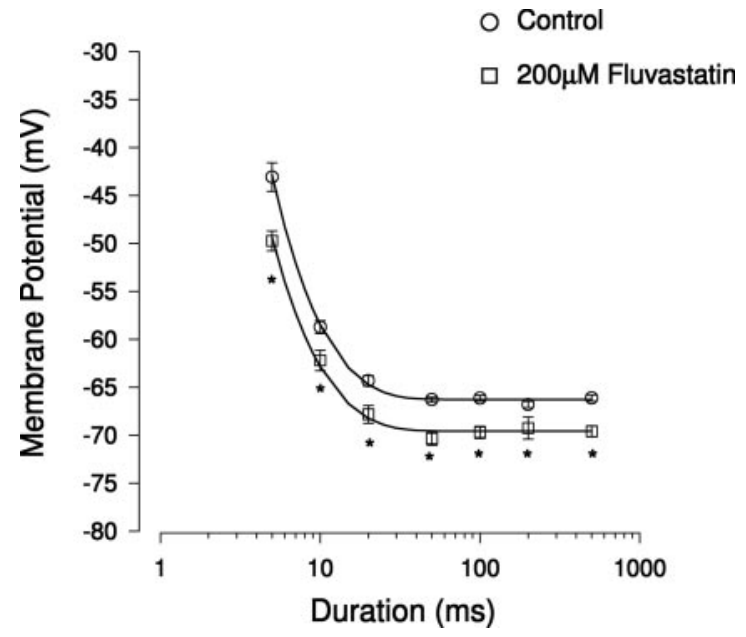

Fig. 5. MT for contraction of EDL muscle fibers from untreated rats before and after in vitro application of $200 \mu \mathrm{M}$ fluvastatin. Each point, expressed as mean value \pm S.E.M. from 34 to 61 fibers of three rats, shows the voltage potential for fiber contraction at each pulse duration. The curves fitting the experimental points have been obtained using the equation described under Materials and Methods. *, significantly more negative with respect to control value (by Student's $t$ test, $P<0.05$ ). 


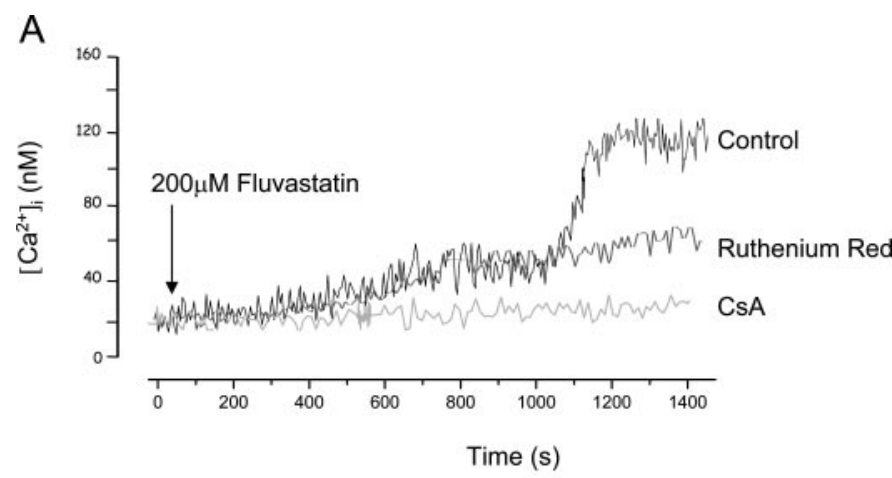

B

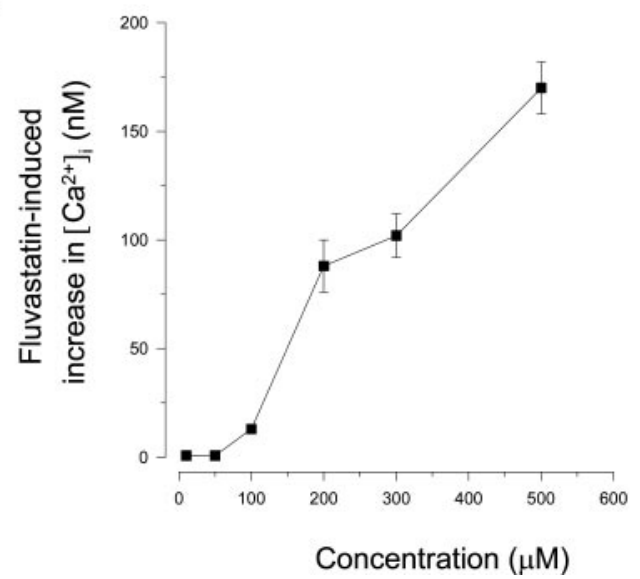

Fig. 6. Effect of in vitro application of fluvastatin on $\left[\mathrm{Ca}^{2+}\right]_{\mathrm{i}}$ of EDL muscle fibers of untreated rats. A, superimposed traces showing the effect of the application of $200 \mu \mathrm{M}$ fluvastatin in control condition (black trace) in the presence of the ryanodine receptor antagonist ruthenium red (RR) (gray trace) or in the presence of the mitochondrial PTP inhibitor CsA (light gray trace). Particularly, muscle fibers were incubated with RR for $5 \mathrm{~min}$ and with CsA for $10 \mathrm{~min}$ before fluvastatin application. B, doseresponse relationship for the effect of fluvastatin on $\left[\mathrm{Ca}^{2+}\right]_{i}$. Data are shown as mean \pm S.E.M., and each point is representative of 15 to 24 fibers.

slight increase of $\left[\mathrm{Ca}^{2+}\right]_{\mathrm{i}}$. Therefore, in further investigations, we routinely applied fluvastatin at $200 \mu \mathrm{M}$.

$\mathrm{Mn}^{2+}$ quench rate. In agreement with the reduction of the cation membrane permeability observed in $20 \mathrm{mg} \mathrm{kg}^{-1}$ day $^{-1}$ fluvastatin-treated animals, in vitro application of fluvastatin reduced fluorescence quench. Particularly, the mean quench rate was $3.9 \pm 0.15$ and $2.89 \pm 0.16 \% \mathrm{~min}^{-1}$ before and after application of $200 \mu \mathrm{M}$ fluvastatin, respectively.

It has been proposed that store-operated calcium entry (SOCE) is essential for maintenance of $\mathrm{Ca}^{2+}$ homeostasis by ensuring the refilling of intracellular calcium storage (Kurebayashi and Ogawa, 2001; Zhao et al., 2005, 2006). Thus, a reduction of SOCE activity could account for the reduced caffeine or potassium-induced calcium transient observed in muscle fibers after in vivo treatment with $20 \mathrm{mg} \mathrm{kg}^{-1} \mathrm{day}^{-1}$ fluvastatin.

To confirm this hypothesis, we evaluated the effect of fluvastatin on $\mathrm{Mn}^{2+}$ permeability after SOCE activation. To this end, $\mathrm{SR} \mathrm{Ca}^{2+}$ stores of muscle fibers were depleted by treatment with thapsigargin and caffeine (Kurebayashi and Ogawa, 2001; Zhao et al., 2006; Liantonio et al., 2007).

As shown in Fig. 7A, activation of SOCE induced by SR $\mathrm{Ca}^{2+}$ depletion led to a steep $\mathrm{Mn}^{2+}$ quenching of fura-2 fluorescence. Interestingly, we found that the preincubation
A
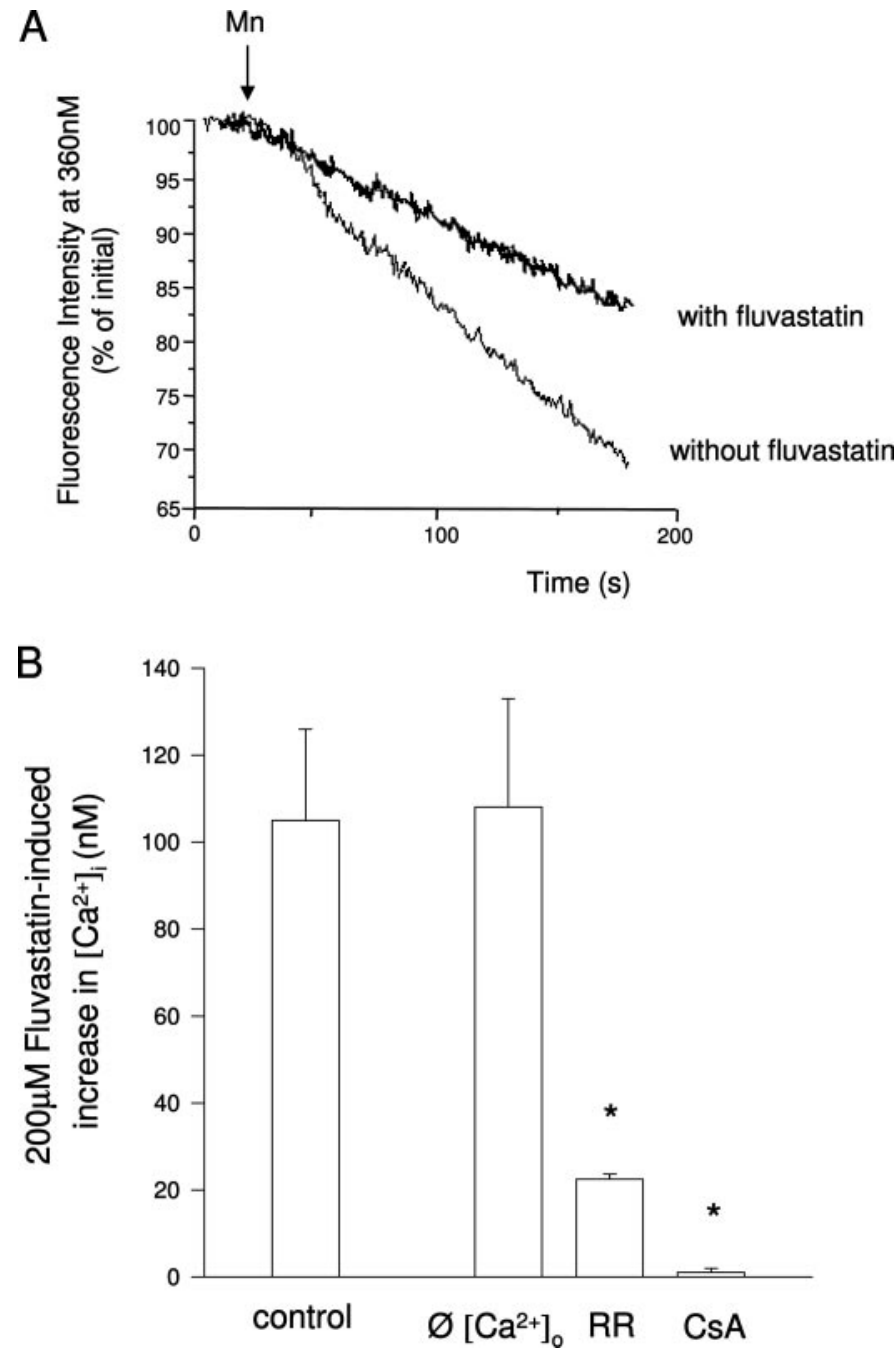

Fig. 7. A, effect of in vitro application of fluvastatin on store-operated calcium entry. Traces represent fura-2 fluorescence quench associated with $\mathrm{Mn}^{2+}$ influx in skeletal muscle fibers following depletion of $\mathrm{SR} \mathrm{Ca}^{2+}$ stores induced by thapsigargin and caffeine (see Materials and Methods) in the absence and presence of fluvastatin. Muscle fibers were treated with thapsigargin/caffeine in zero calcium solution for $15 \mathrm{~min}$ in the absence or presence of fluvastatin, followed by $\mathrm{Mn}^{2+}$ addition to the perfusate. $\mathrm{B}$, investigation of the calcium source responsible of the fluvastatin-induced increase of $\left[\mathrm{Ca}^{2+}\right]_{i}$. The increase in $\left[\mathrm{Ca}^{2+}\right]_{i}$ induced by $200 \mu \mathrm{M}$ fluvastatin measured in the presence of external calcium (control, $n=16)$, in the absence of external calcium $\left(\varnothing \mathrm{Ca}^{2+}, n=15\right)$, or in the presence of $\mathrm{RR}(n=17)$ or $\mathrm{CsA}(n=15)$ was reported. Each bar represents the mean \pm S.E.M. *, significantly different with respect to control value with $P<0.05$ (by Student's $t$ test).

of muscle fibers with $200 \mu \mathrm{M}$ fluvastatin significantly reduced the rate of $\mathrm{Mn}^{2+}$ entry following depletion of $\mathrm{SR} \mathrm{Ca}^{2+}$ store (Fig. 7A). Indeed, the mean quench rate resulted in $9.1 \pm 0.15(n=15)$ and $5.1 \pm 0.12 \% \min ^{-1}(n=13)$ in nontreated and fluvastatin-treated muscle fibers, respectively.

Investigation of the source of calcium release. Removal of external $\mathrm{Ca}^{2+}$ in the bath solution did not abolish the fluvastatin-induced $\left[\mathrm{Ca}^{2+}\right]_{\mathrm{i}}$ rise, producing an increase of $108 \pm$ $22 \mathrm{nM}$, a value not significantly different from that obtained in the presence of extracellular calcium (Fig. 7B). Together with the lack of $\mathrm{Mn}^{2+}$ permeability enhancement after fluvastatin in vitro application (see above), this result strongly suggested that fluvastatin induced a $\left[\mathrm{Ca}^{2+}\right]_{\mathrm{i}}$ increase proba- 
bly by favoring a calcium release from intracellular stores. Thus, we attempted to identify the relevant intracellular source. We investigated the possible involvement of SR using ruthenium red, an inhibitor of the $\mathrm{SR} \mathrm{Ca}^{2+}$ release channels (RyR). Pretreatment with ruthenium red, at a concentration (5 $\mu \mathrm{M}$ ) that completely prevented the $40 \mathrm{mM}$ caffeine effect (Liantonio et al., 2007), significantly reduced the fluvastatininduced $\left[\mathrm{Ca}^{2+}\right]_{i}$ increase, although a $\left[\mathrm{Ca}^{2+}\right]_{i}$ increase of $22.5 \pm 1.2 \mathrm{nM}$ was still observed. (Figs. 6A and 7B). These data indicated that fluvastatin mobilized $\mathrm{Ca}^{2+}$ mainly from SR but that another intracellular calcium store was involved. It is well known that mitochondria play a role in skeletal muscle $\left[\mathrm{Ca}^{2+}\right]_{\mathrm{i}}$ (Saris and Carafoli, 2005). To assess a possible mitochondrial origin of the fluvastatin-induced $\left[\mathrm{Ca}^{2+}\right]_{i}$ increase, we evaluated the effect of such statin in the presence of CsA, an inhibitor of the mitochondrial permeability transition pore (PTP) (Crompton et al., 1999; Liantonio et al., 2007).

In agreement with previous study (Liantonio et al., 2007), the application of $2 \mu \mathrm{M}$ CsA did not alter the baseline of $\left[\mathrm{Ca}^{2+}\right]_{\mathrm{i}}$ (data not shown). Successive addition of $200 \mu \mathrm{M}$ fluvastatin failed to produce any $\left[\mathrm{Ca}^{2+}\right]_{i}$ increase (Fig. 7B). Thus, the inhibition of the mitochondrial PTP completely abolished the fluvastatin-induced $\left[\mathrm{Ca}^{2+}\right]_{\mathrm{i}}$ increase.

\section{Discussion}

The etiology of statin-induced skeletal muscle damage is still under debate. Considering the great number of statintreated patients, the comprehension of mechanisms of statininduced myotoxicity is a determinant in order to prevent these adverse effects. Calcium ions exert a pivotal role in regulating muscle physiology (Berchtold et al., 2000), and an alteration of calcium homeostasis frequently results in muscle damage. Accordingly, elevated resting $\left[\mathrm{Ca}^{2+}\right]_{\mathrm{i}}$ levels were found in pathophysiological conditions of skeletal muscle, such as ageing (Navarro et al., 2001; Fraysse et al., 2006) or muscular dystrophy (Fraysse et al., 2004; De Luca et al., 2003). In the present study, we investigated the potential relationship between statin myotoxicity and calcium homeostasis by assessing the effects of a chronic treatment with fluvastatin and atorvastatin on fura-2-loaded rat intact skeletal muscle fibers.

Either $5 \mathrm{mg} \mathrm{kg}^{-1}$ day $^{-1}$ fluvastatin or atorvastatin at both doses produced a significant increase in $\left[\mathrm{Ca}^{2+}\right]_{i}$, with a concomitant decrease of the caffeine responsiveness and no changes in sarcolemmal calcium permeability. These findings clearly indicated a drug capability of altering calcium homeostasis by interfering with intracellular targets involved with calcium handling mechanisms. It is noteworthy that $20 \mathrm{mg} \mathrm{kg}^{-1}$ day $^{-1}$ fluvastatin-treated animals, in addition to a marked increase of $\left[\mathrm{Ca}^{2+}\right]_{i}$, showed a completely compromised sarcomere organization and a significant decrease of the depolarization-induced intracellular calcium peak. It might be possible that, at this high drug dosage together with the disruption of calcium homeostasis, a series of other cellular mechanisms may take place, all events accounting for the described detrimental effects. An interesting hypothesis could be that fluvastatin administration would produce an alteration of the T-tubule membrane composition. Indeed, the T-tubule membrane system of striated muscle cells was highly enriched in cholesterol (Rosemblatt et al.,
1981), and an alteration of the amount of this component caused a reduction of the depolarization-induced muscle tension (Launikonis and Stephenson, 2001). In support of this hypothesis, it was recently reported that skeletal muscle biopsies from statin-treated patients are characterized by a breakdown of the T-tubular system and by subsarcolemmal ruptures (Draeger et al., 2006).

The degree of calcium homeostasis impairment induced by the statin administration well correlated with the in vivo muscle function performance alteration. Indeed, rats treated with $20 \mathrm{mg} \mathrm{kg}^{-1} \mathrm{day}^{-1}$ fluvastatin showed a reduced force production after prolonged activity, thus indicating a fatiguerelated decrease in muscle function of this animal group with respect to control rats.

To define the statin cellular mechanism of action, leading to calcium homeostasis alteration, an in vitro study was conducted. It is noteworthy that micromolar acute application of fluvastatin caused changes of $\left[\mathrm{Ca}^{2+}\right]_{\mathrm{i}}$, resembling the effect obtained after the in vivo administration. Indeed, fluvastatin was capable of producing a shift of MT for contraction toward more negative potentials and an increase of resting $\left[\mathrm{Ca}^{2+}\right]_{\mathrm{i}}$. Furthermore, in vitro fluvastatin application reduced the cation membrane permeability at rest, an effect that resulted particularly markedly when SOCE was activated. These results allowed us to explain some of the statininduced effects observed after in vivo treatment. Indeed, it was recently demonstrated that, in skeletal muscle, SOCE is essential for the maintenance of $\mathrm{Ca}^{2+}$ homeostasis by ensuring the refilling of intracellular calcium storage (Kurebayashi and Ogawa, 2001; Zhao et al., 2005, 2006). The direct statin SOCE inhibition would lead to a chronic depletion of the intracellular stores, thus justifying the drastic reduction of the potassium- and caffeine-induced $\mathrm{Ca}^{2+}$ transients, characterizing the muscle fibers of $20 \mathrm{mg} \mathrm{kg}^{-1}$ day $^{-1}$ fluvastatin-treated animals. The in vivo and in vitro characterization of the type of the voltage-insensitive permeable calcium current, effectively involved in the SOCE-related effect induced by statins, will be very useful to define the role and the relevance of such phenomenon in mediating the statin-induced myotoxicity.

Accordingly, to quench measurements performed on both in vivo and in vitro statin-treated fibers, the amplitude of the fluvastatin-induced increase of $\left[\mathrm{Ca}^{2+}\right]_{\mathrm{i}}$ did not vary after withdrawal of extracellular calcium, thus strongly indicating that the drug effect on resting $\left[\mathrm{Ca}^{2+}\right]_{i}$ is not due to an increase of the sarcolemmal cationic permeability but rather to an internal $\mathrm{Ca}^{2+}$ store depletion.

By using appropriate tools, we determined the sequence of the statin-induced $\mathrm{Ca}^{2+}$ release mechanism. Mitochondria appeared as the cellular structure responsible for the earlier event, leading to a sustained $\left[\mathrm{Ca}^{2+}\right]_{\mathrm{i}}$ elevation. Indeed, the partial and the complete lack of effect of fluvastatin observed in the presence of ruthenium red and CsA, respectively, led us to propose that a mitochondrial $\mathrm{Ca}^{2+}$ efflux represents the early event, which in turn caused the subsequent large SR $\mathrm{Ca}^{2+}$ release. It is noteworthy that our findings represent the first direct evidence of such mechanism on intact skeletal muscle fibers where the e-c coupling system functionality is ensured and are in agreement with previous studies performed on cultured myoblasts as well as on skinned fibers (Nakahara et al., 1994; Inoue et al., 2003; Sirvent et al., 2005). 
To support direct activity of fluvastatin on mitochondria, it was reported that various lipophilic statins produced alterations in mitochondrial function associated with dissipation of the electric potential across the inner mitochondrial membrane (Kaufmann et al., 2006; Velho et al., 2006), an event that could account for the opening of PTP observed in our investigations. Furthermore, tight structural association between the SR and mitochondria has been described in many cell types (Rizzuto et al., 1998), and recently, a close functional connection between the two cellular structures has been reported in adult fast- and slow-twitch skeletal muscle (Shkryl and Shirokova, 2006). The statin-induced mitochondrial $\mathrm{Ca}^{2+}$ efflux, albeit of small entity, might be highly localized near SR microdomains in close contact with mitochondria, sufficiently resulting in activating $\mathrm{Ca}^{2+}$-induced $\mathrm{Ca}^{2+}$ release from SR. Nevertheless, we cannot exclude the hypothesis that the $\mathrm{Ca}^{2+}$ movement triggered by the opening of PTP might activate a successive adequate cellular signal, which in turn could cause a direct stimulation of calcium efflux from SR.

It is noteworthy that the in vitro reported mitochondrial calcium efflux could allow us also to explain the alteration of caffeine responsiveness observed after in vivo administration. Indeed, because the RyR channel activity was depressed through direct $\mathrm{Ca}^{2+}$-dependent regulation (Fill and Copello, 2002), the reduced caffeine responsiveness, particularly marked for $20 \mathrm{mg} \mathrm{kg}^{-1}$ day $^{-1}$ fluvastatin-treated animals, could probably be due to an alteration of these SR calcium release channels, other than SOCE inhibition, consequent to the persistent mitochondria-derived $\mathrm{Ca}^{2+}$ stimulation. Finally, we did not exclude that a reduced electrochemical driving force due to the increased cytosolic calcium amount could account for the limited caffeine effect.

All our data support the finding that statins induced an alteration in $\mathrm{Ca}^{2+}$ homeostasis in skeletal muscle fibers. The effects on $\mathrm{Ca}^{2+}$ signaling elicited by statins on vascular smooth muscle (de Sotomayor et al., 2005) and on endothelial cells (O’Meara and Kinsella, 2004; Heinke et al., 2004) explain some of the beneficial cardiovascular effects of HMGCoA reductase inhibitors that are not related to their lipidlowering properties. In contrast, such an effect on skeletal muscle could cause various cell damages strongly contributing to the pathogenesis of myopathy induced by statins. The most probable way that elevation in free cytosolic $\mathrm{Ca}^{2+}$ acts to disrupt excitation-contraction coupling in skeletal muscle is via stimulation of certain $\mathrm{Ca}^{2+}$-activated enzymes, such as calpains, phospholipase $\mathrm{A}_{2}$, or protein kinase $\mathrm{C}$. In this regard, it will be highly interesting to investigate whether the statin-induced increase in resting $\left[\mathrm{Ca}^{2+}\right]_{\mathrm{i}}$ may contribute to the decrease of gCl (Pierno et al., 1995, 2006) through a calcium-dependent protein kinase C-dependent CLC-1 inhibition. This may allow a better understanding of the crosstalk mechanism between the two main early targets, cytosolic calcium level and macroscopic gCl, most probably contributing to statin-induced myotoxicity (Pierno et al., 2006).

In conclusion, herein we give the first evidence that an increase of $\left[\mathrm{Ca}^{2+}\right]_{\mathrm{i}}$ in intact tendon-to-tendon rat skeletal muscle fibers can be obtained upon in vivo treatment with fluvastatin or atorvastatin. This effect was attributable to a $\mathrm{Ca}^{2+}$ release from $\mathrm{SR}$ and mitochondria. Considering the crucial role of resting calcium ions in skeletal muscle func- tion and maintenance, our findings considerably contribute to the understanding of the myopathic mechanism induced by this widely used class of hypolipidemic drugs.

\section{Acknowledgments}

We thank Jean-Francois Desaphy and Jean-Francois Rolland for suggestions and comments during the experimental work.

\section{References}

Bellosta S, Paoletti R, and Corsini A (2004) Safety of statins: focus on clinical pharmacokinetics and drug interactions. Circulation 109:50-57.

Berchtold MW, Brinkmeier H, and Muntener M (2000) Calcium ion in skeletal muscle: its crucial role for muscle function, plasticity, and disease. Physiol Rev 80:1215-1265.

Crompton M, Virji S, Doyle V, Johnson N, and Ward JM (1999) The mitochondrial permeability transition pore. Biochem Soc Symp 66:167-179.

De Luca A Pierno S, Liantonio A Camerino C, and Conte Camerino D (1998) Phosphorylation and IGF-1-mediated dephosphorylation pathways control the activity and the pharmacological properties of skeletal muscle chloride channels. Br J Pharmacol 125:477-482.

De Luca A, Pierno S, Liantonio A, Cetrone M, Camerino C, Fraysse B, Mirabella M, Servidei S, Ruegg UT, and Conte Camerino D (2003) Enhanced dystrophic progression in mdx mice by exercise and beneficial effects of taurine and insulin-like growth factor-1. J Pharmacol Exp Ther 304:453-463.

de Sotomayor MA, Perez-Guerrero C, Herrrera MD, Jimenez L, Marin R, Marhuenda $\mathrm{E}$, and Andriantsitohaina R (2005) Improvement of age-related endothelial dysfunction by simvastatin: effect on NO and COX pathways. $\mathrm{Br} J$ Pharmacol 146: $1130-1138$.

Draeger A, Monastyrskaya K, Mohaupt M, Hoppeler H, Savolainen H, Allemann C and Babiychuk E (2006) Statin therapy induces ultrastructural damage in skeletal muscle in patients without myalgia. J Pathol 210:94-102.

Dulhunty AF (1992) The voltage-activation of contraction in skeletal muscle. Prog Biophys Mol Biol 57:181-223.

Evans M and Rees A (2002) The myotoxicity of statins. Curr Opin Lipidol 13:415420

Fill M and Copello JA (2002) Ryanodine receptor calcium release channels. Physiol Rev 82:893-922.

Fraysse B, Desaphy JF, Pierno S, De Luca A, Liantonio A, Mitolo CI, and Conte Camerino D (2003) Decrease in resting calcium and calcium entry associated with slow-to-fast transition in unloaded rat soleus muscle. FASEB J 17:1916-1918.

Fraysse B, Liantonio A, Cetrone M, Burdi R, Pierno S, Frigeri A, Pisoni M, Camerino $\mathrm{C}$, and De Luca A (2004) The alteration of calcium homeostasis in adult dystrophic mdx muscle fibers is worsened by a chronic exercise in vivo. Neurobiol Dis 17: $144-154$.

Fraysse B, Desaphy JF, Rolland JF, Pierno S, Liantonio A, Giannuzzi V, Camerino C, Didonna MP, Cocchi D, De Luca A, et al. (2006) Fiber type-related changes in rat skeletal muscle calcium homeostasis durino aging and restoration by growth hormone. Neurobiol Dis 21:372-380.

Goldstein JL and Brown MS (1990) Regulation of the mevalonate pathway. Nature (Lond) 343:425-430

Grynkiewicz G, Poenie M, and Tsien RY (1985) A new generation of $\mathrm{Ca}^{2+}$ indicators with greatly improved fluorescence properties. $J$ Biol Chem 260:3440-3450.

Heinke S, Schwarz G, Figulla HR, and Heinemann SH (2004) The influence of statins on the free intracellular calcium concentration in human umbilical vein endothelial cells. BMC Cardiovasc Disord 4:4.

Inoue R, Tanabe M, Kono K, Maruyama K, Ikemoto T, and Endo M (2003) $\mathrm{Ca}^{2+}$. releasing effect of cerivastatin on the sarcoplasmic reticulum of mouse and rat skeletal muscle fibers. J Pharmacol Sci. 93:279-288.

Kaufmann P, Torok M, Zahno A, Waldhauser KM, Brecht K, and Krahenbuhl S (2006) Toxicity of statins on rat skeletal muscle mitochondria. Cell Mol Life Sc 63:2415-2425.

Kurebayashi N and Ogawa Y (2001) Depletion of $\mathrm{Ca}^{2+}$ in the sarcoplasmic reticulum stimulates $\mathrm{Ca}^{2+}$ entry into mouse skeletal muscle fibres. J Physiol (Lond) 533: 185-199.

Launikonis BS and Stephenson DG (2001) Effects of membrane cholesterol manipulation on excitation-contraction coupling in skeletal muscle of the toad. J Physiol (Lond) 534:71-85

Liantonio A, Giannuzzi V, Picollo A, Babini E, Pusch M, and Conte Camerino D (2007) Niflumic acid inhibits chloride conductance of rat skeletal muscle by directly inhibiting the CLC-1 channel and by increasing intracellular calcium. $\mathrm{Br} J$ Pharmacol 150:235-247.

Nakahara K, Yada T, Kuriyama M, and Osame M (1994) Cytosolic $\mathrm{Ca}^{2+}$ increase and cell damage in L6 rat myoblasts by HMG-CoA reductase inhibitors. Biochem Biophys Res Commun 202:1579-1585.

Navarro A, Lopez-Cepero JM, and Sanchez del Pino MJ (2001) Skeletal muscle and aging. Front Biosci 6:D26-D44.

O'Meara SJ and Kinsella BT (2004) Effect of the statin atorvastatin on intracellular signalling by the prostacyclin receptor in vitro and in vivo. $\mathrm{Br} J$ Pharmacol 143:292-302

Parekh AB and Penner R (1997) Store depletion and calcium influx. Physiol Rev 77:901-930.

Pierno S, De Luca A, Tricarico D, Roselli A, Natuzzi F, Ferrannini E, Laico M, and Camerino DC (1995) Potential risk of myopathy by HMG-CoA reductase inhibitors: a comparison of pravastatin and simvastatin effects on membrane electrical properties of rat skeletal muscle fibers. J Pharmacol Exp Ther 275:1490-1496.

Pierno S, De Luca A, Liantonio A, Camerino C, and Conte Camerino D (1999) Effects 
of HMG-CoA reductase inhibitors on excitation-contraction coupling of rat skeletal muscle. Eur J Pharmacol 364:43-48.

Pierno S, Didonna MP, Cippone V, De Luca A, Pisoni M, Frigeri A, Nicchia GP, Svelto M, Chiesa G, Sirtori C, et al. (2006) Evaluation of the effects of statins and fibrate chronic treatment on rat skeletal muscle: a biochemical, histological and electrophysiological study. Br J Pharmacol 149:909-919.

Rios E and Pizarro G (1991) Voltage sensor of excitation-contraction coupling in skeletal muscle. Physiol Rev 71:849-908.

Rizzuto R, Pinton P, Carrington W, Fay FS, Fogarty KE, Lifshitz LM, Tuft RA, and Pozzan T (1998) Close contacts with the endoplasmic reticulum as determinants of mitochondrial $\mathrm{Ca}^{2+}$ responses. Science (Wash DC) 280:1763-1766.

Rosemblatt M, Hidalgo C, Vergara C, and Ikemoto N (1981) Immunological and biochemical properties of transverse tubule membranes isolated from rabbit skel etal muscle. J Biol Chem 256:8140-8148.

Rosenbohm A, Rudel R, and Fahlke C (1999) Regulation of the human skeletal muscle chloride channel hClC-1 by protein kinase C. J Physiol (Lond) 514:677-685

Rosenson RS (2004) Statins: can the new generation make an impression? Expert Opin Emerg Drugs 9:269-279.

Sacher J, Weigl L, Werner M, Szegedi C, and Hohenegger M (2005) Delineation of myotoxicity induced by 3-hydroxy-3-methylglutaryl CoA reductase inhibitors in human skeletal muscle cells. J Pharmacol Exp Ther 314:1032-1034.
Saris NE and Carafoli E (2005) A historical review of cellular calcium handling, with emphasis on mitochondria. Biochemistry (Moscow) 70:187-194.

Shkryl VM and Shirokova N (2006) Transfer and tunneling of $\mathrm{Ca}^{2+}$ from sarcoplasmic reticulum to mitochondria in skeletal muscle. J Biol Chem 281:1547-1554

Sirvent P, Mercier J, Vassort G, and Lacampagne A (2005) Simvastatin triggers mitochondria-induced $\mathrm{Ca}^{2+}$ signaling alteration in skeletal muscle. Biochem Biophys Res Commun 329:1067-1075.

Velho JA, Okanobo H, Degasperi GR, Matsumoto MY, Alberici LC, Cosso RG Oliveira HC, and Vercesi AE (2006) Statins induce calcium-dependent mitochondrial permeability transition. Toxicology 219:124-132.

Zhao X, Weisleder N, Han X, Pan Z, Parness J, Brotto M, and Ma J (2006) Azumolene inhibits a component of store-operated calcium entry coupled to the skeletal muscle ryanodine receptor. J Biol Chem 281:33477-33486.

Zhao X, Yoshida M, Brotto L, Takeshima H, Weisleder N, Hirata Y, Nosek TM, Ma J, and Brotto M (2005) Enhanced resistance to fatigue and altered calcium handling properties of sarcalumenin knockout mice. Physiol Genomics 23:72-78.

Address correspondence to: Prof. Diana Conte Camerino, Section of Pharmacology, Dept. of Pharmacobiology, Faculty of Pharmacy, University of Bari, Via Orabona, 4, I-70125 Bari, Italy. E-mail: conte@farmbiol.uniba.it 